\title{
EFFECTS OF STIMULATION INTENSITY, GENDER AND HANDEDNESS UPON AUDITORY EVOKED POTENTIALS
}

\author{
SUSANA CAMPOSANO *-FERNANDO LOLAS *
}

\begin{abstract}
SUMMARY - Left handers and women show less anatomical brain asymmetry, larger corpus callosum and more bilateral representation of specific functions. Sensory and cognitive components of cortical auditory evoked potentials (AEP) have been shown to be asymmetric in right handed males and to be influenced by stimulus intensity. In this study the influence of sex, handedness and stimulus intensity upon AEP components is investigated under basal conditions of passive attention. 14 right handed males, 14 right handed females, 14 left handed males, and 14 left handed females were studied while lying awake and paying passive attention to auditory stimulation (series of 100 binaural clicks, duration $1 \mathrm{msec}$, rate $1 / \mathrm{sec}$, at four intensities). $\mathrm{Cz}, \mathrm{C} 3$ and $\mathrm{C} 4$ referenced to linked mastoids and right EOG were recorded. Analysis time was $400 \mathrm{msec}$, average evoked potentials were based on 100 clicks. Stimulus intensity and gender affect early sensory components (P1N1 and N1P2) at central leads, asymmetry is influenced only by handedness, right handers showing larger P1N1 amplitudes over the right hemisphere.
\end{abstract}

KEY WORDS: auditory evoked potential, sex difference, handedness difference.

Efectos de la intensidad de la estimulación, sexo y manualidade sobre el potencial evocado auditivo.

RESƯMEN - Los sujetos zurdos y de sexo femenino tienden a exhibir menores grados de asimetria anatómica, mayores diámetros del cuerpo calloso y mayor representación bilateral de funciones especificas. Los componentes sensoriales y cognitivos del potencial evocado auditivo (PEA) cortical han demostrado sensibilidad a la intensidad de estimulación y asimetría en sujetos masculinos. En este estudio se investiga la influencia de sexo, manualidad e intensidad de la estimulación sobre los componentes corticales del PEA. 56 sujetos voluntarios fueron sometidos a estimulación auditiva (series de 100 clicks binaurales, duración de $1 \mathrm{mseg}$, frecuencia de $1 / \mathrm{seg}$, a cuatro diferentes intensidades) instruyéndoseles a prestar atención pasiva. Se registró derivaciones $\mathrm{Cz}, \mathrm{C3}$ y $\mathrm{C} 4$ referidas a mastoides bilateral y electrooculograma derecho, el tiempo de análisis fue de $400 \mathrm{mseg}$, los promedios fueron obtenidos sobre 100 clicks. La intensidad de la estimulación y el sexo afectan los componentes sesoriales corticales P1N1 y N1P2 en la derivación central. La asimetria s6lo es influenciada por la manualidad; los Ss diestros exhiben mayores amplitudes P1N1 en el hemisferio derecho. nualidad.

PALAVRAS LLAVE: potencial evocado auditivo, diferencia sexual, diferencia de ma-

PALAVRAS CHAVE: potencial evocado auditivo, diferença sexual, diferença manualidade.

Behavioral and physiological hemispheric asymmetry of function has been related to organism variables such as sex and handedness. Left-handers and women tend to show less anatomical asymmetry 5,8,13. The corpus callosum is larger in both groups, in men at the splenium and in left handers at the central region 7,10,16. Early

* Psychophysiology Unit, Department of Physiology and Biophysics, Faculty of Medicine, University of Chile. This reselarch is supported by FONDECYT Project 1126/90, and the University of Chile (D.T.I.).

Dr. Fernando Lolas - Department of Physiology and Biophysics, Faculty of Medicine, University of Chile - P.O. Box 70005 - Santiago 7 - Chile. 
sensory components of cortical auditory potential (AEP) have been shown to be asymmetric in right handed males and to be influenced by stimulus intensity 4,11.

It is investigated in this study the influence of sex, handedness and stimulus intensity upon AEP components and their hemispheric asymmetry in basal conditions. Symmetrical (binaural) stimulation with low informational content (click) and passive attention conditions were used in order to avoid selecting a lateralized processing mode.

\section{METHODS}

52 unpaid volunteers without neurological or hearing impairment were recruited from the university community (14 right handed men, 14 left handed men, 14 right handed women and 14 left handed women). Handedness was assessed using some items of the Edinburgh Inventory 12. Concordant manual preference for writing, eating with a spoon, holding a hammer to hit a nail, and throwing a ball was requested (Ss. who showed mixed preference were not included). For right handers it was also requested not to have a history of dislexia or stuttering and no left handed relatives.

Subjects lying in an acoustically and electrically isolated room were instructed to stay awake and listen to auditory stimulation. Stimulation consisted of four series of 100 binaural clicks, $1 \mathrm{msec}$ in duration, rate $1 / \mathrm{sec}$, at four intensities $(63,74,87$ and $97 \mathrm{~dB}$ SPL). Intensity sequence was random.

Recordings were made from $\mathrm{Cz}, \mathrm{C} 3$ and $\mathrm{C} 4$ referenced to linked mastoids with surface silver-silverchloride electrodes. Right EOG was simultaneously recorded from infra and supraorbital electrodes. Signals were amplified 20,000 times and band pass filtered between 1 and $100 \mathrm{~Hz}$ using Biodata PA 400 amplifiers. Averages were based on 100 clicks at each intensity, without artifact rejection; analysis time was $400 \mathrm{msec}$ spanned by 640 data points.

Table 1 - Amplitude $(u V)$ of P1N1, N1P2 and P2N2 components at CZ.

\begin{tabular}{|c|c|c|c|c|c|c|c|c|}
\hline \multicolumn{9}{|c|}{ P1N1 } \\
\hline & 63 & $\mathbf{d B}$ & 74 & $\mathrm{~dB}$ & 87 & $\mathbf{d B}$ & 97 & $\mathbf{d B}$ \\
\hline Right Handers & $\mathbf{x}$ & $(\mathrm{SD})$ & $\mathbf{x}$ & (SD) & $\mathbf{X}$ & (SD) & $\mathrm{X}$ & $(\mathrm{SD})$ \\
\hline Men $\quad(N=14)$ & 3.97 & (1.69) & 4.27 & (2.41) & 5.30 & (2.60) & 5.15 & $(2.10)$ \\
\hline Women $(N=14)$ & 4.26 & (1.12) & 5.09 & $(2.47)$ & 4.18 & (1.93) & 5.18 & (2.15) \\
\hline \multicolumn{9}{|l|}{ Left Handers } \\
\hline Men $\quad(\mathrm{N}=14)$ & 3.02 & $(1.58)$ & 4.18 & (1.92) & 4.47 & $(1.79)$ & 5.08 & (2.16) \\
\hline Women $(N=14)$ & 3.54 & $(1.39)$ & 3.86 & $(1.50)$ & 3.71 & $(2.27)$ & 5.51 & $(2.30)$ \\
\hline \multicolumn{9}{|c|}{ N1P2 } \\
\hline & 63 & $\mathrm{~dB}$ & 74 & $\mathrm{~dB}$ & 87 & $\mathrm{~dB}$ & 97 & $\mathbf{d B}$ \\
\hline Right Handers & $\mathbf{x}$ & $(\mathrm{SD})$ & $\mathbf{x}$ & $(\mathrm{SD})$ & $\mathbf{X}$ & (SD) & $\mathbf{X}$ & $(\mathrm{SD})$ \\
\hline Men $\quad(N=14)$ & 5.86 & $(2.64)$ & 7.36 & (2.53) & 8.38 & $(3.27)$ & 8.41 & $(3.25)$ \\
\hline Women $(N=14)$ & 7.43 & (2.11) & 7.50 & $(2.09)$ & 8.04 & $(2.90)$ & 8.57 & $(3.10)$ \\
\hline \multicolumn{9}{|l|}{ Left Handers } \\
\hline Men $\quad(N==14)$ & 4.17 & (1.52) & 6.35 & (1.98) & 6.60 & $(2.28)$ & 7.84 & (2.15) \\
\hline Women $(\mathrm{N}=14)$ & 6.57 & $(2.36)$ & 6.50 & (2.73) & 7.78 & (3.35) & 9.61 & $(3.19)$ \\
\hline \multicolumn{9}{|c|}{ P2N2 } \\
\hline & 63 & $d B$ & 74 & $\mathbf{d B}$ & 87 & $\mathrm{~dB}$ & 97 & $\mathrm{~dB}$ \\
\hline Right Handers & $\mathbf{x}$ & $(\mathrm{SD})$ & $\mathbf{x}$ & $(\mathrm{SD})$ & $\mathbf{x}$ & (SD) & $\mathbf{x}$ & (SD) \\
\hline Men $\quad(\mathrm{N}=14)$ & 8.06 & (2.91) & 7.28 & (1.99) & 8.26 & (2.23) & 7.33 & $(2.56)$ \\
\hline Women $(N=14)$ & 8.06 & (3.96) & 9.68 & (3.59) & 10.25 & $(4.26)$ & 10.05 & (4.55) \\
\hline \multicolumn{9}{|l|}{ Left Handers } \\
\hline Men $\quad(N=14)$ & 5.22 & $(2.03)$ & 7.40 & (3.39) & 6.26 & (4.24) & 7.27 & $(4.24)$ \\
\hline Women $(\mathrm{N}=14)$ & 6.94 & (2.12) & 6.33 & (1.71) & 8.40 & (2.97) & 9.31 & (2.98) \\
\hline
\end{tabular}


Latency of $\mathrm{P1}, \mathrm{N} 1, \mathrm{P2}$ and $\mathrm{N2}$ components and peak to peak ampliutdes of P1N1, N1P2 and P2N2 complexes were measured using a cursor program. If the maximal excursion at the EOG exceeded maximal peak to peak amplitude at $\mathrm{Cz}$, averages were rejected. Components were identified according to maximal deflection in the following time windows: $\mathrm{P} 1,40-80 \mathrm{~ms} ; \mathrm{N} 1,60-120 \mathrm{~ms} ; \mathrm{P} 2,100-190 \mathrm{~ms} ; \mathrm{N} 2,150-300 \mathrm{~ms}$.

An asymmetry coefficient for the proportional activation of the left hemisphere (Left Proportional Amplitude Coefficient, LPAC) was obtained as follows: LPAC=.Amplitude at C3/Ampl. at C3 + Ampl. at C4.

Amplitude and latency averages are shown in Tables 1 and 2. Analysis of variance (ANOVA, Balanced) was performed for $\mathrm{Cz}$ latencies and amplitudes and for LPAC, factors being sex, handedness and intensity. $\mathrm{C3}$ and $\mathrm{C4}$ amplitude and latency differences were analyzed using nonparametric procedures (Wilcoxon Sign Rank Test).

Table 2 - Latency (msec) of P1, N1, P2, and N2 components at CZ.

\begin{tabular}{|c|c|c|c|c|c|c|c|c|}
\hline & & & P1 & & & & & \\
\hline & 63 & $d B$ & 74 & $\mathbf{d B}$ & 87 & $\mathbf{d B}$ & 97 & $\mathbf{d B}$ \\
\hline Right Handers & $\mathbf{x}$ & (SD) & $\mathbf{X}$ & (SD) & $\mathbf{X}$ & $(\mathrm{SD})$ & $\mathbf{x}$ & (SD) \\
\hline Men $\quad(N=14)$ & 54.2 & $(7.1)$ & 53.5 & $(9.2)$ & 54.6 & $(9.2)$ & 52.1 & (3.9) \\
\hline Women $(N=14)$ & 55.6 & $(6.3)$ & 58.4 & $(9.0)$ & 56.4 & (4.9) & 52.5 & $(5.5)$ \\
\hline \multicolumn{9}{|l|}{ Left Handers } \\
\hline Men $\quad(N=14)$ & 55.8 & $(6.5)$ & 55.9 & $(4.3)$ & 55.2 & $(4.1)$ & 54.9 & $(4.5)$ \\
\hline Women $(\mathrm{N}=14)$ & 56.9 & $(7.6)$ & 57.7 & (10.8) & 56.1 & $(8.7)$ & 56.9 & $(8.0)$ \\
\hline \multicolumn{9}{|c|}{ N1 } \\
\hline & 63 & $\mathbf{d B}$ & 74 & $\mathbf{d B}$ & 87 & $\mathbf{d B}$ & 97 & $d B$ \\
\hline Right Hanaer & $\mathbf{x}$ & (SD) & $\mathbf{X}$ & (SD) & $\mathbf{X}$ & (SD) & $\mathbf{X}$ & (SD) \\
\hline Men $\quad(\mathrm{N}=14)$ & 83.9 & $(7.4)$ & 80.4 & $(9.8)$ & 80.4 & (8.9) & 79.1 & (9.3) \\
\hline Women $\quad(N==14)$ & 84.8 & (8.9) & 85.2 & $(12.0)$ & 85.0 & (11.3) & 81.2 & (10.8) \\
\hline \multicolumn{9}{|l|}{ Left Handers } \\
\hline Men $\quad(\mathrm{N}=14)$ & 88.6 & (10.6) & 88.5 & (12.3) & 83.6 & (11.1) & 82.4 & (12.9) \\
\hline Women $(N=14)$ & 89.0 & (12.7) & 80.1 & (10.7) & 79.2 & $(7.5)$ & 80.9 & (12.4) \\
\hline \multicolumn{9}{|c|}{ P2 } \\
\hline & 63 & $\mathbf{d B}$ & 74 & $\mathrm{~dB}$ & 87 & $\mathbf{d B}$ & 97 & $\mathbf{d B}$ \\
\hline Right Handers & $\mathbf{X}$ & (SD) & $\mathbf{X}$ & (SD) & $\mathbf{X}$ & (SD) & $\mathbf{X}$ & (SD) \\
\hline Men $\quad(\mathrm{N}=14)$ & 144.5 & (10.2) & 146.1 & (12.6) & 145.0 & (13.9) & 144.0 & (10.5) \\
\hline Women $(N==14)$ & 141.6 & (15.7) & 147.4 & (11.3) & 146.4 & (11.3) & 141.2 & (10.1) \\
\hline \multicolumn{9}{|l|}{ Left Handers } \\
\hline Men $\quad(\mathrm{N}=14)$ & 155.5 & (16.7) & 153.4 & (19.6) & 148.9 & (17.2) & 147.7 & (16.2) \\
\hline Women $(\mathrm{N}=14)$ & 142.6 & (12.9) & 142.0 & (16.5) & 145.0 & (10.8) & 143.7 & (16.3) \\
\hline \multicolumn{9}{|c|}{ N2 } \\
\hline & 63 & $\mathrm{~dB}$ & 74 & $\mathrm{~dB}$ & 87 & $\mathbf{d B}$ & 97 & $\mathbf{d B}$ \\
\hline Right Handers & $\mathbf{x}$ & (SD) & $\mathbf{x}$ & (SD) & $\mathbf{X}$ & (SD) & $\mathbf{x}$ & (SD) \\
\hline Men $\quad(N=14)$ & 225.5 & (15.5) & 217.5 & $(20.8)$ & 229.7 & (23.3) & 223.1 & (20.1) \\
\hline Women $(\mathrm{N}=14)$ & 220.2 & $(29.0)$ & 240.1 & $(28.0)$ & 223.1 & (22.5) & 231.2 & (26.3) \\
\hline Left Handers & & & & & & & & \\
\hline Men $\quad(N=14)$ & 221.2 & (27.4) & 234.3 & $(28.0)$ & 223.5 & (32.7) & 240.3 & $(27.8)$ \\
\hline Women $(\mathrm{N}=14)$ & 214.1 & (24.1) & 222.3 & $(32.9)$ & 223.9 & (28.6) & 229.4 & (37.2) \\
\hline
\end{tabular}




\section{RESULTS}

A tendency to larger amplitudes in women and right handers can be appreciated in Figure 1. Amplitude and latency averages and standard deviations are shown in Tables 1 and 2.
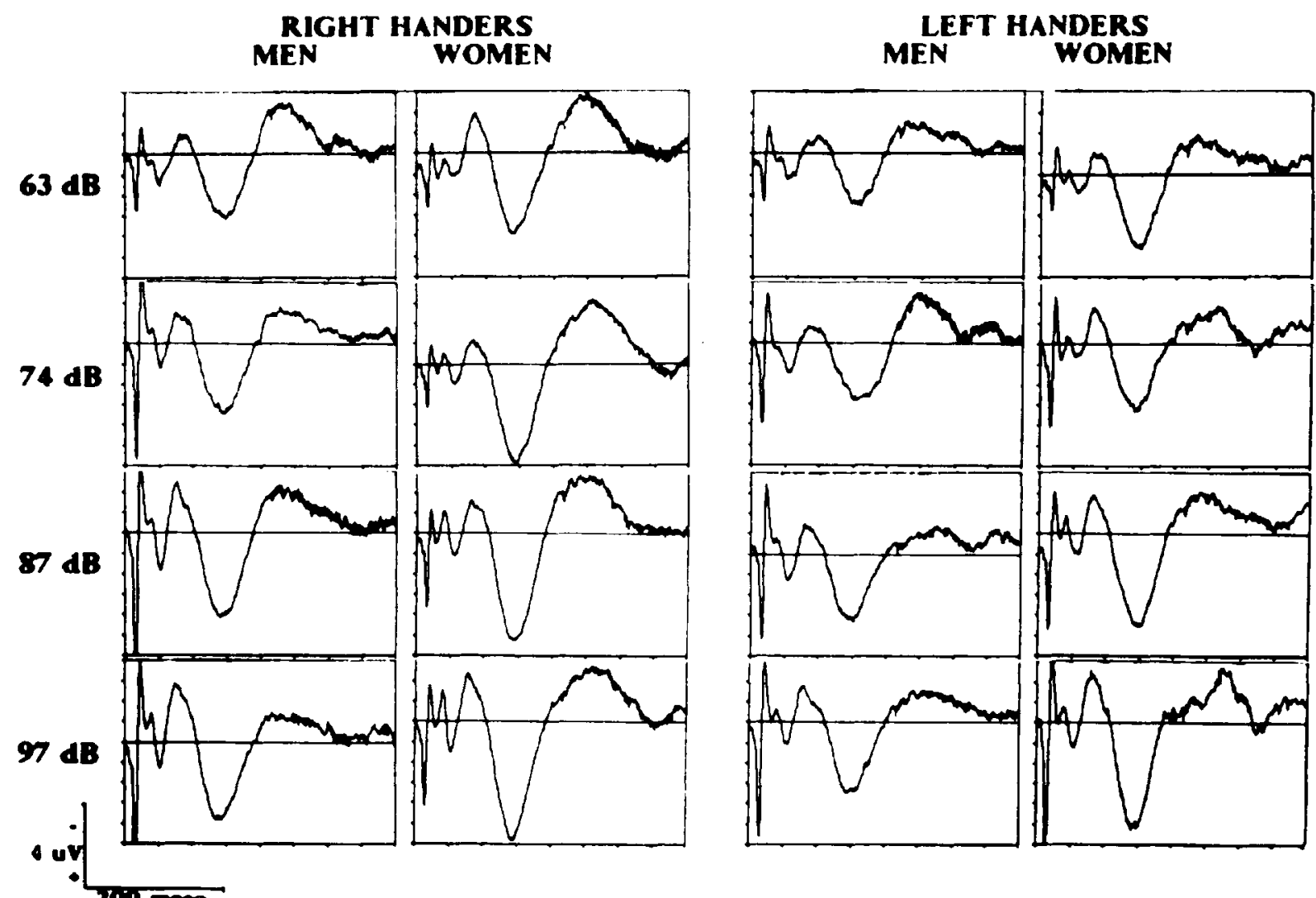

Fig. 1 - Grand averages at $C z$ according to sex and handedness at four intensities. $N=14$ for each average.

Analysis of variance for central lead P1N1, N1P2 and P2N2 peak-to-peak amplitudes shows different influence of the variables studied on the components (Table 3). Latency was less influenced by these factors (Table 4).

Right-left differences in amplitude were found for P1N1 in right handers, Wilcoxon: C4 $>\mathrm{C} 3$ in right handed males at 74 and $87 \mathrm{~dB}(\mathrm{p}<0.05) ; \mathrm{C} 4>\mathrm{C} 3$ in right handed females at $63 \mathrm{~dB}(\mathrm{p}<0.02)$. P1N1 amplitude at $\mathrm{C} 3$ and $\mathrm{C} 4$ is shown in Table 5 .

Amplitude asymmetry as measured by LPAC is only influenced by handedness (Table 6). Values for LPAC of right and left handers are shown in Table 4. Differences can be appreciated in Figure 2. There is no latency asymmetry.

Table 3 - Significant factors affecting components peak to peak amplitwde at $C z$ and their interactions as determined by analysis of variance.

\begin{tabular}{lccc}
\hline Anova & \multicolumn{3}{c}{ Amplitude at Cz } \\
\cline { 3 - 4 } & P1N1 & N1P2 & P2N2 \\
\hline sex & $\mathrm{ns}$ & $\mathrm{p}<0.02$ & $\mathrm{~ns}$ \\
man pref & $\mathrm{ns}$ & $\mathrm{p}<0.05$ & $\mathrm{p}<0.01$ \\
intensity & $\mathrm{p}<0.01$ & $\mathrm{p}<0.001$ & $\mathrm{~ns}$ \\
sex*man pref & $\mathrm{ns}$ & $\mathrm{ns}$ & $\mathrm{ns}$ \\
sex*int & $\mathrm{p}<0.05$ & $\mathrm{p}<0.001$ & $\mathrm{~ns}$ \\
man pref*int & $\mathrm{p}<0.03$ & $\mathrm{p}<0.000$ & $\mathrm{p}<0.03$ \\
sex*man pref*int & $\mathrm{ns}$ & $\mathrm{p}<0.002$ & $\mathrm{~ns}$ \\
\end{tabular}


Table 4 - Significant factors affecting components latency at $C z$ and their interactions as determined by analysis of variance.

\begin{tabular}{|c|c|c|c|c|}
\hline \multirow[t]{2}{*}{ Anova } & \multicolumn{4}{|c|}{ Latency at $\mathrm{Cz}$} \\
\hline & P1 & N1 & P2 & N2 \\
\hline $\operatorname{sex}$ & ns & ns & $\mathrm{p}<0.03$ & ns \\
\hline man pref & ns & ns & $\mathrm{ns}$ & ns \\
\hline intensity & ns & $\mathrm{p}<0.05$ & $\mathrm{~ns}$ & $\mathrm{~ns}$ \\
\hline sex*man plef & $\mathrm{p}<0.03$ & $\mathrm{~ns}$ & ns & $\mathbf{n s}$ \\
\hline sex*int & $\mathrm{ns}$ & $\mathrm{ns}$ & $\mathrm{ns}$ & ns \\
\hline man pref*int & $\mathrm{ns}$ & $\mathrm{ns}$ & $\mathrm{ns}$ & ns \\
\hline sex*man pref*int & ns & ns & ns & ns \\
\hline
\end{tabular}

Table 5-P1N1 amplitude at C3 and C4.

\begin{tabular}{|c|c|c|c|c|c|c|c|c|c|}
\hline & & \multicolumn{4}{|c|}{ C3 } & \multicolumn{4}{|c|}{$\mathrm{C4}$} \\
\hline & & $63 \mathrm{~dB}$ & $74 \mathrm{~dB}$ & $87 \mathrm{~dB}$ & $97 \mathrm{~dB}$ & $63 \mathrm{~dB}$ & $74 \mathrm{~dB}$ & $87 \mathrm{~dB}$ & $97 \mathrm{~dB}$ \\
\hline \multicolumn{10}{|c|}{ Right handers } \\
\hline $\begin{array}{l}\text { Men } \\
(N==14)\end{array}$ & $\begin{array}{c}\mathbf{X} \\
(\mathrm{SD})\end{array}$ & $\begin{array}{c}3.50 \\
(1.57)\end{array}$ & $\begin{array}{c}3.41 \\
(1.94)\end{array}$ & $\begin{array}{c}4.22 \\
(1.90)\end{array}$ & $\begin{array}{c}3.77 \\
(1.52)\end{array}$ & $\begin{array}{c}4.00 \\
(1.64)\end{array}$ & $\begin{array}{c}4.19 \\
(2.75)\end{array}$ & $\begin{array}{c}4.75 \\
(3.16)\end{array}$ & $\begin{array}{c}4.54 \\
(2.35)\end{array}$ \\
\hline $\begin{array}{l}\text { Women } \\
(\mathrm{N}-=14)\end{array}$ & $\begin{array}{c}X \\
(\mathrm{SD})\end{array}$ & $\begin{array}{c}3.88 \\
(1.33)\end{array}$ & $\begin{array}{c}3.14 \\
(1.08)\end{array}$ & $\begin{array}{c}3.39 \\
(1.47)\end{array}$ & $\begin{array}{c}3.48 \\
(1.94)\end{array}$ & $\begin{array}{c}4.42 \\
(1.02)\end{array}$ & $\begin{array}{c}3.34 \\
(1.16)\end{array}$ & $\begin{array}{c}3.97 \\
(1.61)\end{array}$ & $\begin{array}{c}3.62 \\
(1.68)\end{array}$ \\
\hline \multicolumn{10}{|c|}{ Left handers } \\
\hline $\begin{array}{l}\text { Men } \\
(\mathrm{N}=\mathrm{i} 4)\end{array}$ & $\frac{x}{(S D)}$ & $\begin{array}{c}3.05 \\
(2.02)\end{array}$ & $\begin{array}{c}3.79 \\
(1.89)\end{array}$ & $\begin{array}{c}3.93 \\
(1.37)\end{array}$ & $\begin{array}{c}4.93 \\
(1.55)\end{array}$ & $\begin{array}{c}3.35 \\
(1.50)\end{array}$ & $\begin{array}{c}3.77 \\
(1.70)\end{array}$ & $\begin{array}{c}3.66 \\
(1.95)\end{array}$ & $\begin{array}{c}4.02 \\
(2.49)\end{array}$ \\
\hline $\begin{array}{l}\text { Women } \\
(\mathrm{N}=-14)\end{array}$ & $\begin{array}{c}X \\
(\mathrm{SD})\end{array}$ & $\begin{array}{c}3.77 \\
(1.41)\end{array}$ & $\begin{array}{c}3.79 \\
(1.37)\end{array}$ & $\begin{array}{c}3.93 \\
(1.78)\end{array}$ & $\begin{array}{r}4.93 \\
(1.87)\end{array}$ & $\begin{array}{c}3.55 \\
(1.45)\end{array}$ & $\begin{array}{c}3.80 \\
(1.68)\end{array}$ & $\begin{array}{c}4.24 \\
(1.84)\end{array}$ & $\begin{array}{c}4.75 \\
(1.57)\end{array}$ \\
\hline
\end{tabular}

Table 6 - Significant factors affecting asymmetry of components peak to peak amplitude, represented in the left proportional amplitude coefficient (LPAC), as determined by analysis of variances.

\begin{tabular}{llccc}
\hline Anova of LPAC & P1N1 & N1P2 & P2N2 \\
\hline sex & $\mathrm{ns}$ & $\mathrm{ns}$ & $\mathrm{ns}$ \\
man pref & $<0.004$ & $\mathrm{~ns}$ & $\mathrm{p}<0.001$ \\
intensity & $\mathrm{ns}$ & $\mathrm{ns}$ & $\mathrm{ns}$ \\
sex*man pref & $\mathrm{ns}$ & $\mathrm{ns}$ & $\mathrm{ns}$ \\
sex*int & $\mathrm{ns}$ & $\mathrm{ns}$ & $\mathrm{ns}$ \\
man pref*int & $\mathrm{ns}$ & $\mathrm{ns}$ & $\mathrm{ns}$ \\
sex*man pref*int & $\mathrm{ns}$ & $\mathrm{ns}$ & $\mathrm{ns}$
\end{tabular}




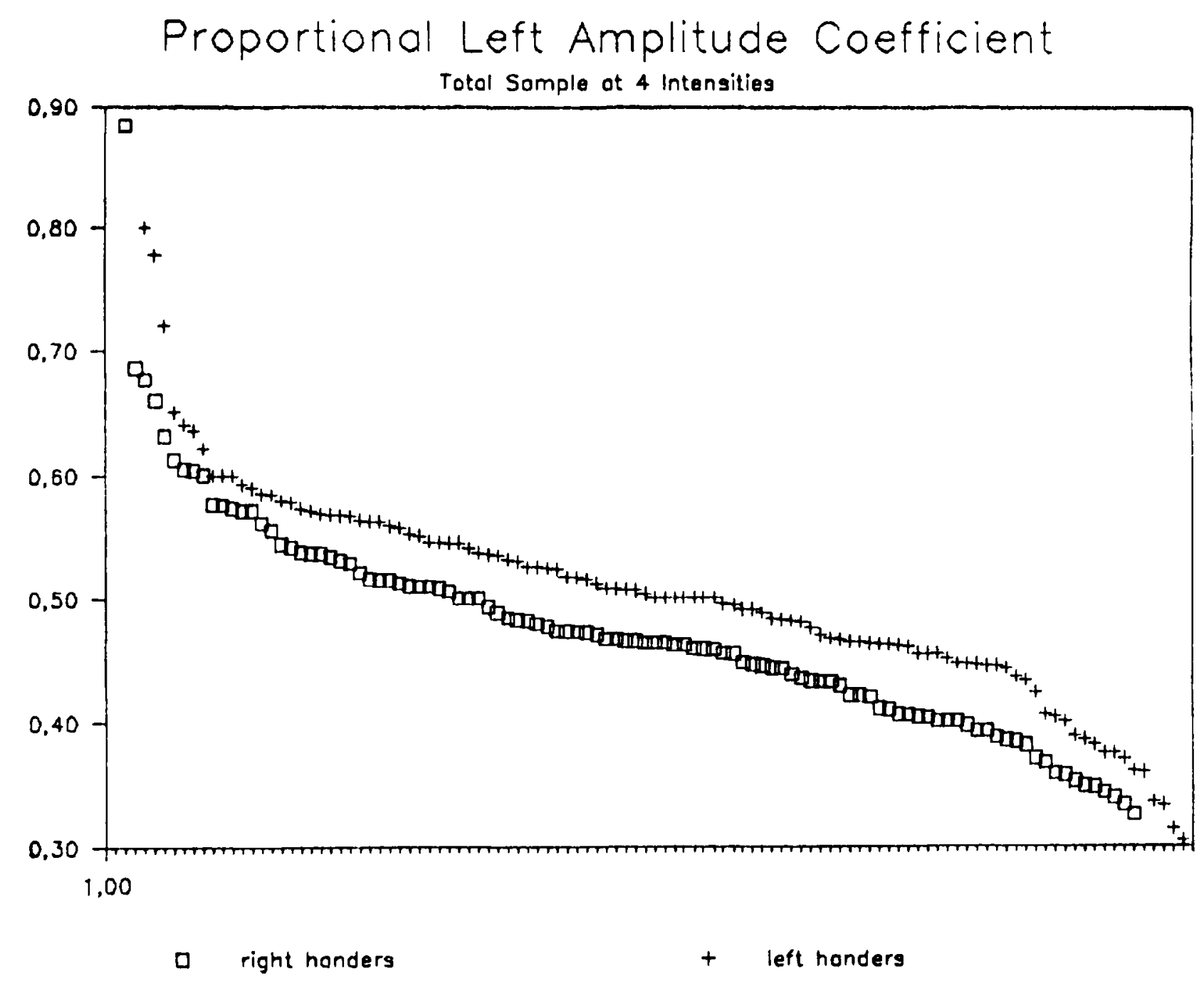

Fig. 2 - Left proportional amplitude coefficient (LPAC=C3 ampl./C3+C4ampl.) for $P 1 N 1$ in right and left handers at four intensities. $N=56$.

\section{COMMENTS}

Stimulus intensity and gender influence early sensory components of AEP at central leads but exert no influence on hemispheric asymmetry of electrocortical signals. Asymmetry is influenced only by handedness, with right handers showing larger amplitudes over the right hemisphere.

Amplitude at central lead is influenced by the three factors studied: P1N1 by intensity; N1P2 by intensity, gender and handedness; and P2N2 only by handedness. The influence of intensity on early cortical components is well known, therefore they are called «stimulus dependent» 14. Sexual differences are also known, women tend to have larger amplitudes 3 .

The influence of handedness on central lead amplitude is a new finding and could be explained if left handers had more bilateral activation of supratemporal regions. Only right handed subjects show greater $\mathrm{C} 4$ amplitudes, in left handers no significant asymmetry could be demonstrated. Amplitude depends on previous activation of generator sites, the greater the previous activation, the lower is the evoked amplitude 15. This view would be concordant with greater left side activation in right handers (thus showing lower amplitudes at C3) and with bilateral activation in left handers (thus showing less asymmetry and lower amplitudes at $\mathrm{Cz}$ ). This bilateral activation can be related to the thicker central part of corpus callosum, which includes auditory cortex callosal fibers 1 . Anova shows asymmetry as measured by LPAC of P1N1 and P2N2 to be influenced only by handedness.

This kind of AEP study is complementary with behavioral asymmetry studies and contributes data on early and basic aspects of hemispheric processing, which can be used for prediction of behavioral outcomes. 


\section{REFERENCES}

1. Alexander MD, Warren RL. Localization of callosial auditory pathways: a CT case study. Neurology 1988, 38:802-804.

2. Bear D, Schiff D, Saver J, Greenberg M, Freeman R. Quantitative analysis of cerebral asymmetries. Arch Neurol 1986, 43:598-603.

3. Buchsbaum MS, Henkin RI, Christiansen RL. Age and sex differences in averaged evoked responses in a normal population, with observation of patients with gonadal dysgenesis. Electroenceph Clin Neurophysiol 1974, 37:137-144.

4. Collin C, Lolas F. Hemispheric asymmetry of auditory evoked potential (AEP) augmenting-reducing. Psychophysiology 1983, 20:436-439.

5. Czachowska-Sieszycka B, Szelag E. Hemispheric asymmetry in processing of verbal material by left-handers. Physiologia Bohemoslovaca 1985, 34:21-24.

6. Galaburda AM. Biological correlates of left-handedness. Semin Neurol 1984, 4:120-125.

7. Holloway RL, Lacoste MC. Sexual dimorphism in the human corpus callosum: an extension and replication study. Human Neurobiol 1986, 5:87-91.

8. Kimura D, Harshman R. Sex differences in brain organization for verbal and non verbal functions. Programs Brain Res 1984, 61:423-441.

9. Koff E, Naeser MA, Pieniadz JM, Foundas AL, Levine HL. Computed tomographic scan hemispheric asymmetries in right - and left-handed male and female subjects. Arch Neurol 1986, 43:487-491.

10. Lacoste-Utamsing $\mathbf{C}$, Holloway RL. Sexual dimorphism in the human corpus callosum. Science 1982, 216:1431-1432.

11. Lolas F, Collin C, Camposano S, Etcheberrigaray $R$, Rees $R$. Hemispheric asymmetry of augmenting/reducing in visual and auditory evoked potentials. Biol Psychiat 1987, 22:1413-1416.

12. Oldfield RC. The assessment and analysis of handedness: the Endiburgh Inventory. Neuropsychologia 1971, 9:97-113.

13. Piazza DM. The influence of sex and handedness in the hemispheric specialization of verbal and nonverbal tasks. Neuropsychologia 1980, 18:163-176.

14. Picton TW, Hillyard SA, Krausz HI, Galambos R. Human auditory evoked potentials. I: Evaluation of components. Electroenceph Clin Neuphysiol 1974, 36:179-190.

15. Shucard W, Shucard JL, Thomas DG. Auditory evoked potentials as probes of hemispheric differences in cognitive processing. Science 1977, 179:1295-1297.

16. Witelson SF. The brain connection: the corpus callosum is larger in left-handers. Science 1985, 229:665-668. 